\title{
On Weighted Chebyshev-Type Quadrature Formulas
}

\author{
By Klaus-Jürgen Förster and Georg-Peter Ostermeyer
}

\begin{abstract}
A weighted quadrature formula is of Chebyshev type if it has equal coefficients and real (but not necessarily distinct) nodes. For a given weight function we study the set $T(n, d)$ consisting of all Chebyshev-type formulas with $n$ nodes and at least degree $d$. It is shown that in nonempty $T(n, d)$ there exist two special formulas having "extremal" properties. This result is used to prove uniqueness and further results for $E$-optimal Chebyshev-type formulas. For the weight function $w \equiv 1$, numerical investigations are carried out for $n \leqslant 25$.
\end{abstract}

1. Introduction. Let $w$ be a nonnegative weight function on the interval $(a, b)$, $-\infty \leqslant a<b \leqslant \infty$, admitting moments $m_{j}$ of all order

$$
m_{j}=\int_{a}^{b} x^{j} w(x) d x, \quad j=0,1,2, \ldots, m_{0}>0 .
$$

We consider (weighted) Chebyshev-type quadrature formulas [7]. These are quadrature formulas $Q_{n}$ with equal coefficients and real (but not necessarily distinct) nodes:

$$
\begin{aligned}
Q_{n}[f]:= & c \sum_{i=1}^{n} f\left(x_{i}\right), \quad-\infty<x_{1} \leqslant x_{2} \leqslant \cdots \leqslant x_{n}<\infty, \\
& \int_{a}^{b} f(x) w(x) d x=Q_{n}[f]+R_{n}[f] .
\end{aligned}
$$

By this definition, it is possible that some nodes $x_{i}$ are not contained in the interval $(a, b) . Q_{n}$ has at least degree $d$ (of exactness) if

$$
R_{n}\left[p_{i}\right]=0, \quad i=0,1, \ldots, d,
$$

where $p_{i}$, here and throughout this paper, denotes the monomial $p_{i}(x):=x^{i}$. If $d \geqslant 0$, the coefficient $c$ in (1.2) is determined by (1.3):

$$
c=m_{0} / n \text {. }
$$

The maximal possible degree of a Chebyshev-type quadrature formula with $n$ nodes is denoted by $d_{n}$.

Let, in the following, $T(n, d)$ be the set of all Chebyshev-type quadrature formulas with $n$ nodes and at least degree $d$. One has $T(n, d+1) \subseteq T(n, d)$ and $T(n, d) \subseteq T(k n, d)$ for every $k \in \mathbf{N}$. For $n>2$, a simple calculation shows that

Received March 26, 1984; revised November 30, 1984 and April 29. 1985.

1980 Mathematics Subject Classification. Primary 65D30.

(C1986 American Mathematical Society $0025-5718 / 86 \$ 1.00+\$ .25$ per page 
each set $T(n, 2)$ contains an infinite number of elements. In case of $d_{n} \geqslant n$ the set $T\left(n, d_{n}\right)$ contains only one element, the so-called "Chebyshev quadrature formula in the strict sense". Weight functions $w$ which allow such formulas for every $n \in \mathbf{N}$ are rare [7, p. 109]. In case of $d_{n}<n$ the set $T\left(n, d_{n}\right)$ possibly contains more than one element. To select some of these formulas several criteria can be found in the literature.

From a historical point of view it may be obvious to consider such quadrature formulas $Q_{n}^{\text {opt }} \in T\left(n, d_{n}\right)$, which minimize $\left|R_{n}\left[p_{d_{n}+1}\right]\right|$ among all $Q_{n} \in T\left(n, d_{n}\right)$. Such quadrature formulas are called $E$-optimal [9], [2], [7]. In case of weight functions $w$, which are symmetric with respect to the (finite) interval $(a, b)$, several authors have distinguished between symmetric, i.e., $x_{i}-a=b-x_{n-i+1}$ for $i=$ $1, \ldots, n$, and unsymmetric formulas with regard to $E$-optimality [10], [9], [2]. There is computational evidence that $E$-optimal formulas for symmetric weight functions are indeed symmetric [7, p. 113]. Gautschi and Yanagiwara [9] have shown that symmetry would follow, if $E$-optimal formulas are unique in $T\left(n, d_{n}\right)$. One aim of this paper is to prove the uniqueness of $E$-optimal formulas in general.

Several authors have proposed other criteria to select special Chebyshev-type formulas-necessarily not contained in $T\left(n, d_{n}\right)$ (see, e.g., [7]). Therefore, it may be of interest to study the set $T(n, d)$ in general. If $T(n, d)$ contains more than one element, we show that there exists in $T(n, d)$ an infinite number of formulas which have pairwise distinct nodes. In this case, there also exists in $T(n, d)$ an infinite number of interpolatory quadrature formulas (for definition, see, e.g., [3]). Among these interpolatory quadrature formulas there are two unique formulas which have several "extremal" properties with respect to all other formulas in $T(n, d)$. By proving that the $E$-optimal formula $Q_{n}^{\text {opt }}$ is one of the two extremal formulas in $T\left(n, d_{n}\right)$, we can show various properties of $E$-optimal formulas.

The proofs of all theorems can be found in the supplements section of this issue.

2. $E$-Extremal Formulas. We call a (Chebyshev-type quadrature) formula $Q_{n} \in$ $T(n, d) E$-minimal in $T(n, d)$ and denote it by $Q_{n, d}^{\min }$ if

$$
R_{n, d}^{\min }\left[p_{d+1}\right]=\min \left\{R_{n}\left[p_{d+1}\right] \mid Q_{n} \in T(n, d)\right\} .
$$

Correspondingly, we define $E$-maximal formulas $Q_{n, d}^{\max } \in T(n, d)$ by

$$
R_{n, d}^{\max }\left[p_{d+1}\right]=\max \left\{R_{n}\left[p_{d+1}\right] \mid Q_{n} \in T(n, d)\right\} .
$$

Therefore, the following inequalities are valid for every $Q_{n} \in T(n, d)$ :

$$
Q_{n, d}^{\max }\left[p_{d+1}\right] \leqslant Q_{n}\left[p_{d+1}\right] \leqslant Q_{n, d}^{\min }\left[p_{d+1}\right] .
$$

Formulas with property (2.1) or (2.2) we call $E$-extremal. According to the arguments of Gautschi and Yanagiwara [9] for the existence of $E$-optimal formulas there exist $E$-minimal and $E$-maximal formulas in $T(n, d)$ for all $d$ with

$$
1<d \leqslant d_{n} \text {. }
$$

Remark. In the following we require for $d$ the validity of (2.3), unless noted otherwise.

Our first result is the uniqueness of $E$-extremal formulas in $T(n, d)$. 
THEOREM 1. In $T(n, d)$ there exists only one E-minimal formula $Q_{n, d}^{\min }$ and only one E-maximal formula $Q_{n, d}^{\max }$.

Definition (1.2) allows the possibility that some of the nodes coincide. It can be shown that $E$-extremal formulas have multiple nodes. Moreover, the two $E$-extremal formulas can be characterized by a special arrangement of these multiple nodes. To describe this arrangement we define for every Chebyshev-type quadrature formula $Q_{n}$ the sequence $S\left(Q_{n}\right):=\left(s_{i}\left(Q_{n}\right)\right)_{i=1}^{n-1}$ as follows:

$$
s_{i}\left(Q_{n}\right)= \begin{cases}0, & \text { if } x_{n+1-i} \neq x_{n-i}, \\ 1, & \text { if } x_{n+1-i}=x_{n-i} \text { and } i \text { odd } \\ -1, & \text { if } x_{n+1-i}=x_{n-i} \text { and } i \text { even }\end{cases}
$$

We speak of a change of sign of the sequence $S\left(Q_{n}\right)$ (between $s_{i}\left(Q_{n}\right)$ and $s_{i+l}\left(Q_{n}\right)$ ) if

$$
\begin{aligned}
& \operatorname{sign}\left(s_{i}\left(Q_{n}\right)\right)=-\operatorname{sign}\left(s_{i+l}\left(Q_{n}\right)\right) \neq 0 \\
& s_{i+1}\left(Q_{n}\right)=s_{i+2}\left(Q_{n}\right)=\cdots=s_{i+l-1}\left(Q_{n}\right)=0
\end{aligned}
$$

TheOREM 2. Let $Q_{n}$ be E-extremal in $T(n, d)$. Then $Q_{n}$ has at most d distinct nodes. Moreover,

(i) Let $d<n-1$. A formula $Q_{n}$ is E-extremal in $T(n, d)$ if and only if $S\left(Q_{n}\right)$ has at least $(n-d-1)$ changes of sign. In this case the following holds: If the first nonzero term of $S\left(Q_{n}\right)$ is negative, then $Q_{n}$ is E-minimal. If this term is positive, then $Q_{n}$ is E-maximal. If $S\left(Q_{n}\right)$ has more than $(n-d-1)$ changes of sign, then $Q_{n}$ is E-minimal as well as E-maximal and $T(n, d)$ contains only $Q_{n}$.

(ii) Let $d=n-1$. A formula $Q_{n}$ is E-extremal in $T(n, d)$ if and only if $S\left(Q_{n}\right)$ has at least one nonzero term. If this term is negative, then $Q_{n}$ is $E$-minimal. If this term is positive, then $Q_{n}$ is E-maximal. If $S\left(Q_{n}\right)$ has at least one change of sign, then $Q_{n}$ is $E$-minimal as well as $E$-maximal and $T(n, d)$ contains only $Q_{n}$.

Theorem 2 shows that $E$-extremal formulas are interpolatory quadrature formulas (for definition see, e.g., [3]). The following theorem answers the question for other interpolatory quadrature formulas in $T(n, d)$ and for formulas with pairwise distinct nodes.

THEOREM 3. Let $Q_{n, d}^{\min }$ and $Q_{n, d}^{\max }$ be the E-minimal and the E-maximal formula in $T(n, d)$. Let

$$
r \in\left(R_{n, d}^{\min }\left[p_{d+1}\right], R_{n, d}^{\max }\left[p_{d+1}\right]\right)
$$

Then there exist formulas $\tilde{Q}_{n}$ and $\bar{Q}_{n}$ in $T(n, d)$ with $\tilde{R}_{n}\left[p_{d+1}\right]=\bar{R}_{n}\left[p_{d+1}\right]=r$ and

(i) $\tilde{Q}_{n}$ has pairwise distinct nodes,

(ii) $\bar{Q}_{n}$ has at most $(d+1)$ distinct nodes.

In the case of $d<n-1$, there exists for each such $r$ even an infinite number of formulas with property (i). In the case of $d=n-1$ there exists for each such $r$ only 
one formula $Q_{n} \in T(n, d)$ with $R_{n}\left[p_{d+1}\right]=r$ and this formula has pairwise distinct nodes.

A first justification for the consideration of $E$-extremal formulas is the fact that their first and their $n$th node have extremal properties with respect to all $Q_{n} \in$ $T(n, d)$.

THEOREM 4. Let $x_{i}$ be the nodes of a formula $Q_{n} \in T(n, d)$, which is not E-extremal. Let $x_{i}^{\min }$ and $x_{i}^{\max }$ be the nodes of the E-extremal formulas $Q_{n, d}^{\min }$ and $Q_{n, d}^{\max }$ in $T(n, d)$. Then

(i) $x_{n}^{\min }>x_{n}>x_{n}^{\max }$,

(ii) $(-1)^{d} x_{1}^{\min }>(-1)^{d} x_{1}>(-1)^{d} x_{1}^{\max }$.

Therefore, it is also possible to characterize the $E$-minimal ( $E$-maximal) formula in $T(n, d)$ to be that formula, whose $n$th node has the largest (smallest) value.

Furthermore, Theorem 4 may be helpful for the investigation of the question of whether all nodes of a formula $Q_{n} \in T(n, d)$ are contained in the interval $[a, b]$.

The formulas $Q_{n, d}^{\min }$ and $Q_{n, d}^{\max }$ are defined by the extremal property (2.1) and (2.2) of their remainder with respect to only one function, the monomial $p_{d+1}$. The following theorem shows that these extremal properties remain valid for a wide class of functions, which contains especially all monomials $p_{d+2 k-1}$ for all $k \in \mathbf{N}$.

THEOREM 5. Let $Q_{n, d}^{\min }$ and $Q_{n, d}^{\max }$ be the E-minimal and the E-maximal formula in $T(n, d)$. Then, for all $f \in C^{d+1}, f^{(d+1)} \geqslant 0$, there hold

(i) $R_{n, d}^{\min }[f]=\min \left\{R_{n}[f] \mid Q_{n} \in T(n, d)\right\}$,

(ii) $R_{n, d}^{\max }[f]=\max \left\{R_{n}[f] \mid Q_{n} \in T(n, d)\right\}$.

Another interpretation of Theorem 5 may be of interest:

Let $K_{d+1}$ denote the Peano kernel of degree $d+1$ ([7, p. 112], [3, p. 39]), of a formula $Q_{n}$ in $T(n, d)$ and $K_{d+1}^{\min }$, resp. $K_{d+1}^{\max }$, the Peano kernels of the same degree of the $E$-extremal formulas $Q_{n}^{\min }$ or $Q_{n}^{\max }$ in $T(n, d)$. Theorem 5 implies the inequalities

$$
K_{d+1}^{\min }(x) \leqslant K_{d+1}(x) \leqslant K_{d+1}^{\max }(x)
$$

for all $x \in \mathbf{R}$.

3. E-Optimal Formulas. Our basic result for $E$-optimal formulas is given in the following theorem.

TheOReM 6. Let $n \in \mathbf{N}$ and let $Q_{n}^{\text {opt }}$ be E-optimal. Then $Q_{n}^{\text {opt }}$ is E-extremal in $T\left(n, d_{n}\right)$.

An $E$-optimal formula is therefore $E$-minimal or $E$-maximal in $T\left(n, d_{n}\right)$ and has the corresponding properties given in Section 2.

The first part of Theorem 2 has been proven for $E$-optimal formulas by Anderson and Gautschi [2]. The second part of Theorem 2 reduces all the remaining cases to only two formulas, characterized also by the value of the $n$th node according to 
Theorem 4. Theorem 3 shows, in particular, the impossibility that different $E$-extremal formulas in $T\left(n, d_{n}\right)$ are both $E$-optimal. This answers the question of the uniqueness of $E$-optimal formulas [2], [7].

Corollary 1. Let $n \in \mathbf{N}$. Then there exists one and only one E-optimal formula $Q_{n}^{\text {opt }}$.

Therefore, by the result of Gautschi and Yanagiwara [9] mentioned above, it follows from Corollary 1 that

COROLlaRY 2. Let the weight function $w$ be symmetric with respect to $(a, b)$ and let $n \in \mathbf{N}$. Then $d_{n}$ is odd and the E-optimal formula is symmetric.

Rabinowitz and Richter [11] have shown that $E$-optimal rules minimize $\left|R_{n}\right|$ for formulas (1.2) in special function spaces. With the help of Theorem 5, a different justification for the consideration of $E$-optimal formulas is given by the following theorem.

THEOREM 7. Let $n \in \mathbf{N}$ and $Q_{n}^{\text {opt }}$ be the E-optimal formula and let $f \in C^{d_{n}+1}$, $f^{\left(d_{n}+1\right)} \geqslant 0$. If

$$
\operatorname{sign}\left(R_{n}^{\mathrm{opt}}\left[p_{d_{n}+1}\right]\right)=\operatorname{sign}\left(R_{n}^{\mathrm{opt}}[f]\right)
$$

then

$$
\left|R_{n}^{\mathrm{opt}}[f]\right|=\min \left\{\left|R_{n}[f]\right| \mid Q_{n} \in T\left(n, d_{n}\right)\right\} .
$$

4. Numerical Results for the Weight Function $w \equiv 1$. For the weight function $w \equiv 1$ there exist Chebyshev formulas in the strict sense for $n=1,2, \ldots, 7$ and $n=9$-see, e.g., [7]. The $E$-optimal formulas have been computed by Gautschi and Yanagiwara [9] for $n=8,10,11,13$ and by Anderson and Gautschi [2] for $n=12$, $14,15,16,17$. Anderson [1] has shown that these formulas, except for $n=12$, are definite, i.e., there exists a representation of their remainder term of the form

$$
R_{n}[f]=\frac{R_{n}\left[p_{d_{n}+1}\right]}{\left(d_{n}+1\right) !} f^{\left(d_{n}+1\right)}(\xi)
$$

for every $f \in C^{d_{n}+1}$.

The present authors have computed the $E$-extremal formulas in $T\left(n, d_{n}\right)$ for $n \leqslant 25$ by a different method with the help of Theorem 2 resp. Theorem 3 [6]. The $E$-optimal formulas for $n=18, \ldots, 25$ are given at the end of this section. These formulas are all definite. Theorem 5 implies that every $Q_{n} \in T\left(n, d_{n}\right)$ is also definite for $n \leqslant 25, n \neq 12$; for $n=8,10,11,13$ see Förster [4]. In case of definiteness, the comparison of the coefficients of $f^{\left(d_{n}+1\right)}(\xi)$ in (4.1) between the $E$-minimal and the $E$-maximal formula gives information as to how useful the choice of the $E$-optimal formula is in $T\left(n, d_{n}\right)$. These coefficients are listed in Table 1 . In every case, the $E$-minimal formula is $E$-optimal. The numerical results correspond to the interval of integration $[-1,1]$.

A conclusion of the above theorems is that the results of Gautschi and Monegato [8] and Förster [4] for $n=8,10,11,13$ remain valid for all $n \leqslant 25, n \neq 12$. 
TABLE 1

\begin{tabular}{|c|c|c|c|c|c|}
\hline n & $d_{n}$ & $R_{n, d}^{\min }\left[P_{d_{n}+1}\right]$ & $R_{n, d}^{\max }\left[p_{d_{n}+1}\right]$ & $\frac{R_{n, d}^{\max }\left[p_{d_{n}+1}\right]}{R_{n, d}^{\min }\left[p_{d_{n}+1}\right]}$ & \\
\hline 1 & 1 & 0.667 E 0 & 0.667 E 0 & 1 & definite \\
\hline 2 & 3 & $0.178 \mathrm{E} 0$ & $0.178 \mathrm{E} 0$ & 1 & definite \\
\hline 3 & 3 & $0.667 \mathrm{E}-1$ & $0.667 \mathrm{E}-1$ & 1 & definite \\
\hline 4 & 5 & $0.339 \mathrm{E}-1$ & $0.339 \mathrm{E}-1$ & 1 & definite \\
\hline 5 & 5 & $0.172 \mathrm{E}-1$ & $0.172 \mathrm{E}-1$ & 1 & definite \\
\hline 6 & 7 & $0.102 \mathrm{E}-1$ & $0.102 \mathrm{E}-1$ & 1 & definite \\
\hline 7 & 7 & $0.578 \mathrm{E}-2$ & $0.578 E-2$ & 1 & definite \\
\hline 8 & 7 & $0.202 \mathrm{E}-2$ & $0.541 \mathrm{E}-2$ & 2.68 & definite \\
\hline 9 & 9 & $0.221 \mathrm{E}-2$ & $0.221 \mathrm{E}-2$ & 1 & definite \\
\hline 0 & 9 & $0.119 \mathrm{E}-2$ & $0.153 \mathrm{E}-2$ & 1.29 & definite \\
\hline 1 & 9 & $0.573 \mathrm{E}-3$ & $0.155 \mathrm{E}-2$ & 2.71 & definite \\
\hline 2 & 9 & $0.663 E-4$ & $0.121 \mathrm{E}-2$ & 18.25 & $\begin{array}{l}\mathrm{R}_{12}^{\min } \text { not definite } \\
\mathrm{R}_{12}^{\max } \text { definite }\end{array}$ \\
\hline 13 & 11 & $0.384 \mathrm{E}-3$ & $0.440 \mathrm{E}-3$ & 1.15 & definite \\
\hline 14 & 11 & $0.218 \mathrm{E}-3$ & $0.464 E-3$ & 2.13 & definite \\
\hline 15 & 11 & $0.102 \mathrm{E}-3$ & $0.384 \mathrm{E}-3$ & 3.76 & definite \\
\hline 16 & 11 & $0.407 \mathrm{E}-4$ & $0.352 \mathrm{E}-3$ & 8.65 & definite \\
\hline 17 & 13 & $0.105 \mathrm{E}-3$ & $0.117 \mathrm{E}-3$ & 1.11 & definite \\
\hline 18 & 13 & $0.656 \mathrm{E}-4$ & $0.115 E-3$ & 1.75 & definite \\
\hline 19 & 13 & $0.399 \mathrm{E}-4$ & $0.108 \mathrm{E}-3$ & 2.71 & definite \\
\hline 20 & 13 & $0.198 E-4$ & 0.101 E-3 & 5.10 & definite \\
\hline 21 & 13 & $0.6138-5$ & $0.860 E-4$ & 14.03 & definite \\
\hline 22 & 15 & $0.242 \mathrm{E}-4$ & $0.319 E-4$ & 1.32 & definite \\
\hline 23 & 15 & $0.159 \mathrm{E}-4$ & 0.273 E-4 & 1.72 & definite \\
\hline 24 & 15 & $0.102 \mathrm{E}-4$ & $0.298 E-4$ & 2.92 & definite \\
\hline 25 & 15 & $0.594 \mathrm{E}-5$ & $0.262 \mathrm{E}-4$ & 4.41 & definite \\
\hline
\end{tabular}

CoRollary 3. Let $n \leqslant 25$ and $w \equiv 1$. Let $Q_{n}^{\text {opt }}$ be the E-optimal formula and $Q_{n} \in T\left(n, d_{n}\right)$.

(a) If in (1.1) $b=-a$, then for every $m \in \mathbf{N}$,

$$
0 \leqslant R_{n}^{\mathrm{opt}}\left[p_{m}\right] \leqslant R_{n}\left[p_{m}\right]
$$

(b) If $n \neq 12$ and $f \in C^{d_{n}+1}, f^{\left(d_{n}+1\right)} \geqslant 0$, then

$$
0 \leqslant R_{n}^{\text {opt }}[f] \leqslant R_{n}[f]
$$


Therefore, these $E$-optimal formulas satisfy also every optimality criterion of the form

$$
\min \left\{\sum_{i=d_{n}+1}^{\infty} a_{i}\left(R_{n}\left[p_{i}\right]\right)^{2} \mid Q_{n} \in T\left(n, d_{n}\right)\right\}
$$

with any $a_{i} \geqslant 0[7$, p. 113]. They are, in particular for $n \neq 12$, also optimal in the sense of Sard [7, p. 112], [4].

The E-Optimal Formulas for $w \equiv 1 \quad 18 \leqslant n \leqslant 25$

$n=18$

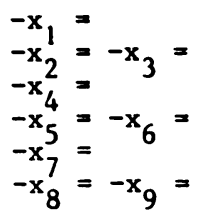

$n=19$

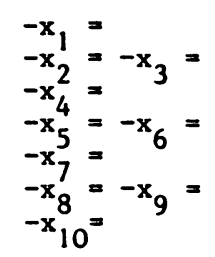

$n=20$

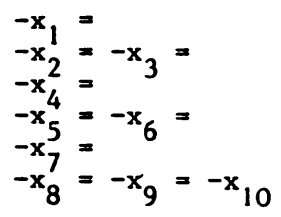

$n=21$

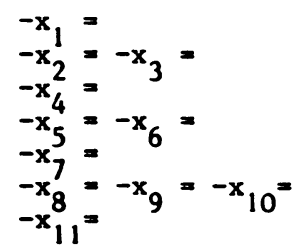

0.95611589370931681977

0.78339593833119703042

0.58679047283945639018

0.45756408008040941541

0.25737493728377540704

0.12068411927871514185

0.95841522638659246454

0.79485226355878236323

0.60772484959475892451

0.48688511013054279206

0.29638895564058655907

0.16315108328419371742

0.0

0.96051482286129288228

0.80496515092537905967

0.63049631592920524269

0.50749481899047359478

0.35906562874648327105

0.15625951409613565727

0.96243015157286074846

0.81403490074542027161

0.65167313907372323093

0.52555764207596964732

0.40559995128245393129

0.18868995126113857540

0.0

$$
\begin{aligned}
& =x_{18} \\
=x_{16} & =x_{17} \\
& =x_{15} \\
=x_{13} & =x_{14} \\
& =x_{12} \\
=x_{10} & =x_{11}
\end{aligned}
$$

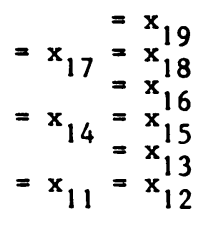


(continued)
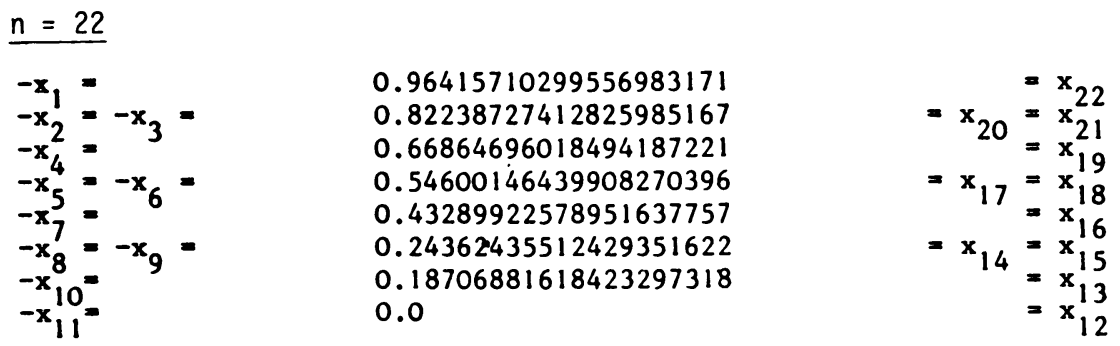

$n=23$

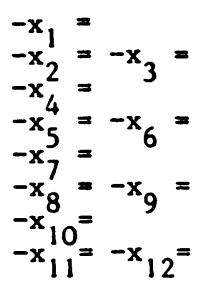

0.96570343338357257096

0.83018849753913168834

0.68178827221824105045

0.56773078130524428871

0.45254730818175202350

0.26810913164371012869

0.25355181302970919482

0.0

$$
\begin{aligned}
& =x_{23} \\
=x_{21} & =x_{22} \\
& =x_{20} \\
=x_{18} & =x_{19} \\
& =x_{17} \\
=x_{15} & =x_{16} \\
& =x_{14} \\
& =x_{13}
\end{aligned}
$$
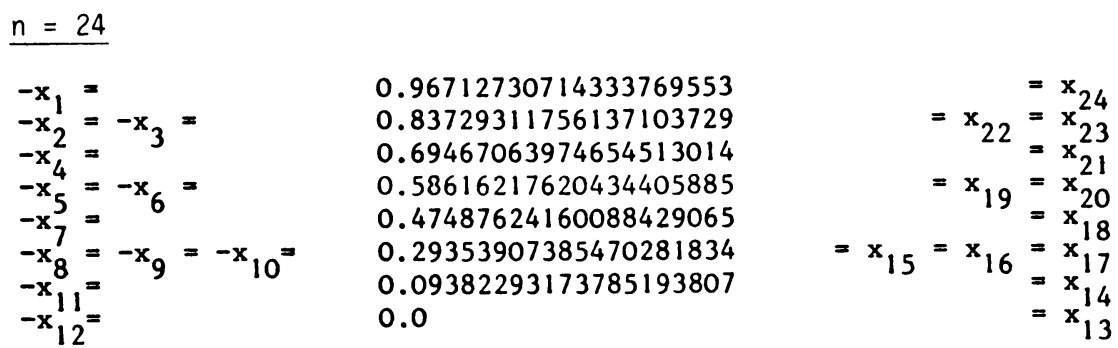

$\underline{n}=25$
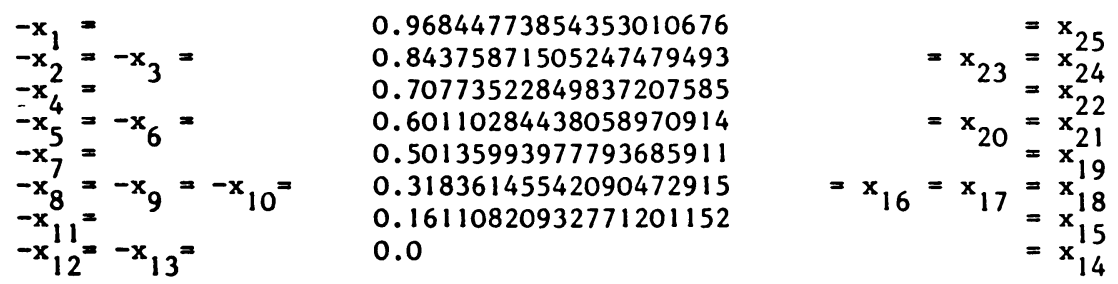

5. Examples. Table 1 shows that in case of $w \equiv 1$ the sets $T\left(n, d_{n}\right)$ for $n \leqslant 25$ and $d_{n}<n$ contain an infinite number of elements (see Theorem 3 ). The same is true for the examples computed by Anderson and Gautschi [2] in case of other weight functions. The following example shows with the help of Theorem 2 the possibility that for $d_{n}<n$ the set $T\left(n, d_{n}\right)$ contains only one element.

Let the weight function $w$ be given by $w(x):=\sqrt{1-x^{2}}$. The corresponding Gauss-formula $G_{5}$ with 5 nodes and therefore degree 9 is given by (see Szegö [12, p. 344])

$$
G_{5}[f]=\frac{\pi}{24}\left\{f\left(-\frac{\sqrt{3}}{2}\right)+3 f\left(-\frac{1}{2}\right)+4 f(0)+3 f\left(\frac{1}{2}\right)+f\left(\frac{\sqrt{3}}{2}\right)\right\} .
$$

Because of $m_{0}=\pi / 2$ the formula $G_{5}$ is a Chebyshev-type quadrature formula (1.2) 
with the twelve nodes

$$
\begin{gathered}
x_{1}=-\frac{\sqrt{3}}{2}=-x_{12}, \\
x_{2}=x_{3}=x_{4}=-\frac{1}{2}=-x_{11}=-x_{10}=-x_{9}, \\
x_{5}=x_{6}=0=-x_{8}=-x_{7} .
\end{gathered}
$$

So $G_{5}$ is an element of $T(12,9)$. By $(2.4)$ the sequence $S\left(G_{5}\right)$ is given by

$$
S\left(G_{5}\right)=(0,1,-1,0,-1,1,-1,0,-1,1,0)
$$

and has four changes of sign; see (2.5). Theorem 2(i) shows that $T(12,9)$ contains only the element $G_{5}$. Furthermore, $G_{5}$ is also the only element of $T(12,8)$, and $G_{5}$ is the $E$-maximal formula $Q_{12,7}^{\max }$ in $T(12,7)$.

In the case of $w \equiv 1$ and $n \leqslant 25$ the nodes of the $E$-minimal formula $Q_{n, d_{n}}^{\min }$ are contained in the interval $(-1,1)$. Therefore, in these cases, using Theorem 4 , the nodes of every formula $Q_{n} \in T\left(n, d_{n}\right)$ are also contained in $(-1,1)$. But this is not so, in general, for every weight function $w$ and every $n \in \mathbf{N}$. The following example shows that there exist even Chebyshev quadrature formulas in the strict sense, i.e., $d_{n} \geqslant n$, with nodes not all contained in $[a, b]$ :

Let $w$ be a weight function on $(-1,1)$ with $w(x):=\left(1-x^{2}\right)^{-4 / 5}$. A simple calculation with the help of Newton's identities (see [7, p. 104]) shows that for $n=3$, 4, 6, 7 the Chebyshev quadrature formulas in the strict sense exist and that their first and last nodes are not contained in $[-1,1]$.

Institut für Angewandte Mathematik

Technische Universität Braunschweig

D-3300 Braunschweig, Federal Republic of Germany

Institut für Technische Mechanik

Technische Universität Braunschweig

D-3300 Braunschweig, Federal Republic of Germany

1. L. A. Anderson, Optimal Chebyshev-Type Quadrature Formulas for Various Weight Functions, Ph. D. Thesis, Purdue University, 1974.

2. L. A. ANDERSON \& W. Gautschi, "Optimal weighted Chebyshev-type quadrature formulas," Calcolo, v. 12, 1975, pp. 211-248.

3. H. BRASS, Quadraturverfahren, Vandenhoeck \& Ruprecht, Göttingen, 1977.

4. K.- J. FORSTER, “Bemerkungen zur optimalen Tschebyscheff-Typ Quadratur,“ Numer. Math., v. 38, 1982, pp. 421-425.

5. K.- J. FORSTER, “A comparison theorem for linear functionals and its application in quadrature," in Numerical Integration (G. Hämmerlin, ed.), (ISNM 57), Birkhäuser Verlag, Berlin, 1982, pp. 66-76.

6. K.- J. FORSTER \& G.-P. OSTERMEYER, Zur Optimalität und Berechnung von Tschebyscheff-TypQuadraturformeln, Bericht 1/1984, Institut für Technische Mechanik der Technischen Universität Braunschweig, 1983.

7. W. Gautschi, Advances in Chebyshev Quadrature, Lecture Notes in Math., vol. 506, Springer-Verlag, Berlin and New York, 1977, pp. 100-121.

8. W. Gautschi \& G. Monegato, “On optimal Chebyshev-type quadratures," Numer. Math., v. 28, 1977, pp. 59-67.

9. W. Gautschi \& H. Yanagiwara, “On Chebyshev-type quadratures,” Math. Comp., v. 28, 1974, pp. 125-134.

10. D. K. Kahaner, “Chebyshev-type quadrature formulas,” Math. Comp., v. 25, 1970, pp. 571-574.

11. P. Rabinowitz \& N. Richter, "Chebyshev-type integration rules of minimum norm," Math. Comp., v. 24, 1970, pp. 831-845.

12. G. Szeg0, Orthogonal Polynomials, Amer. Math. Soc. Colloq. Publ., vol. 23, Amer. Math. Soc., Providence, R.I., 1939. 


\section{Supplement to \\ On Weighted Chebyshev-Type Quadrature Formulas}

\section{By Klaus-Jürgen Förster and Georg-Peter Ostermeyer}

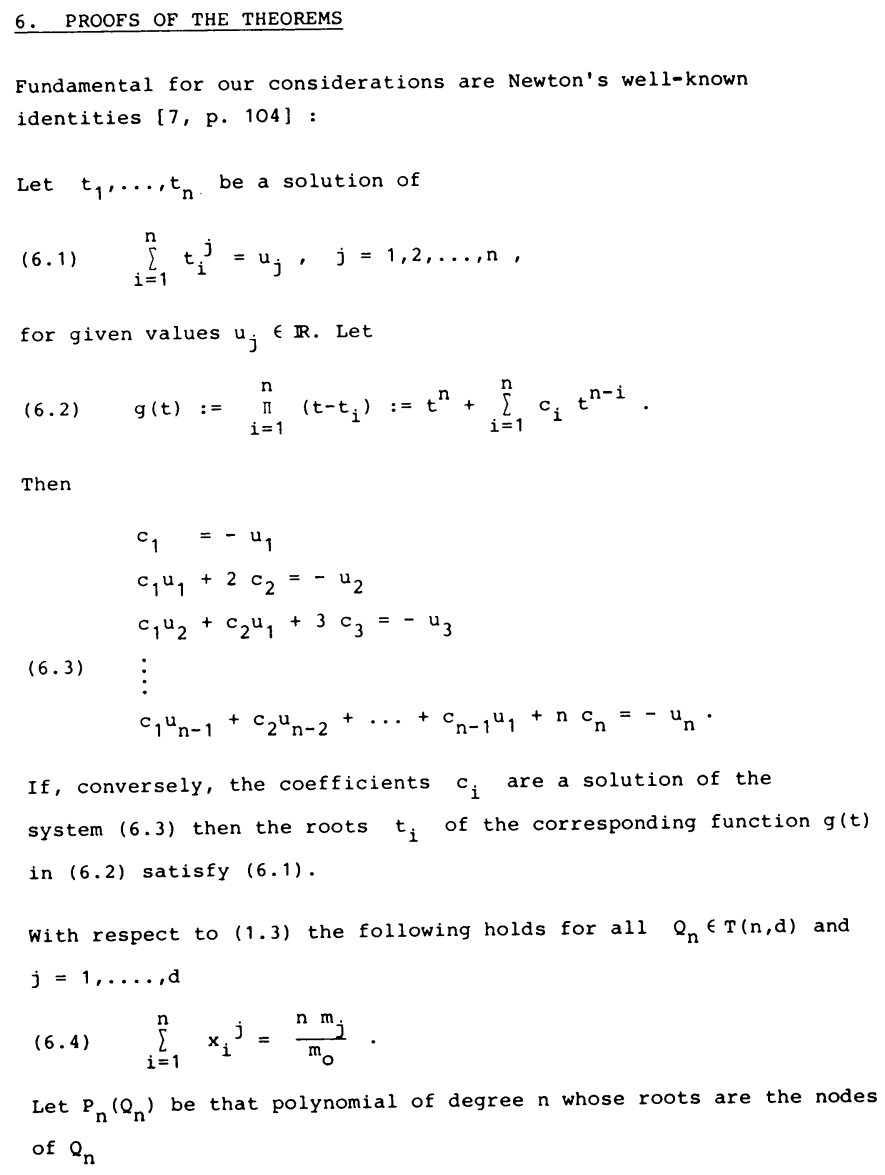



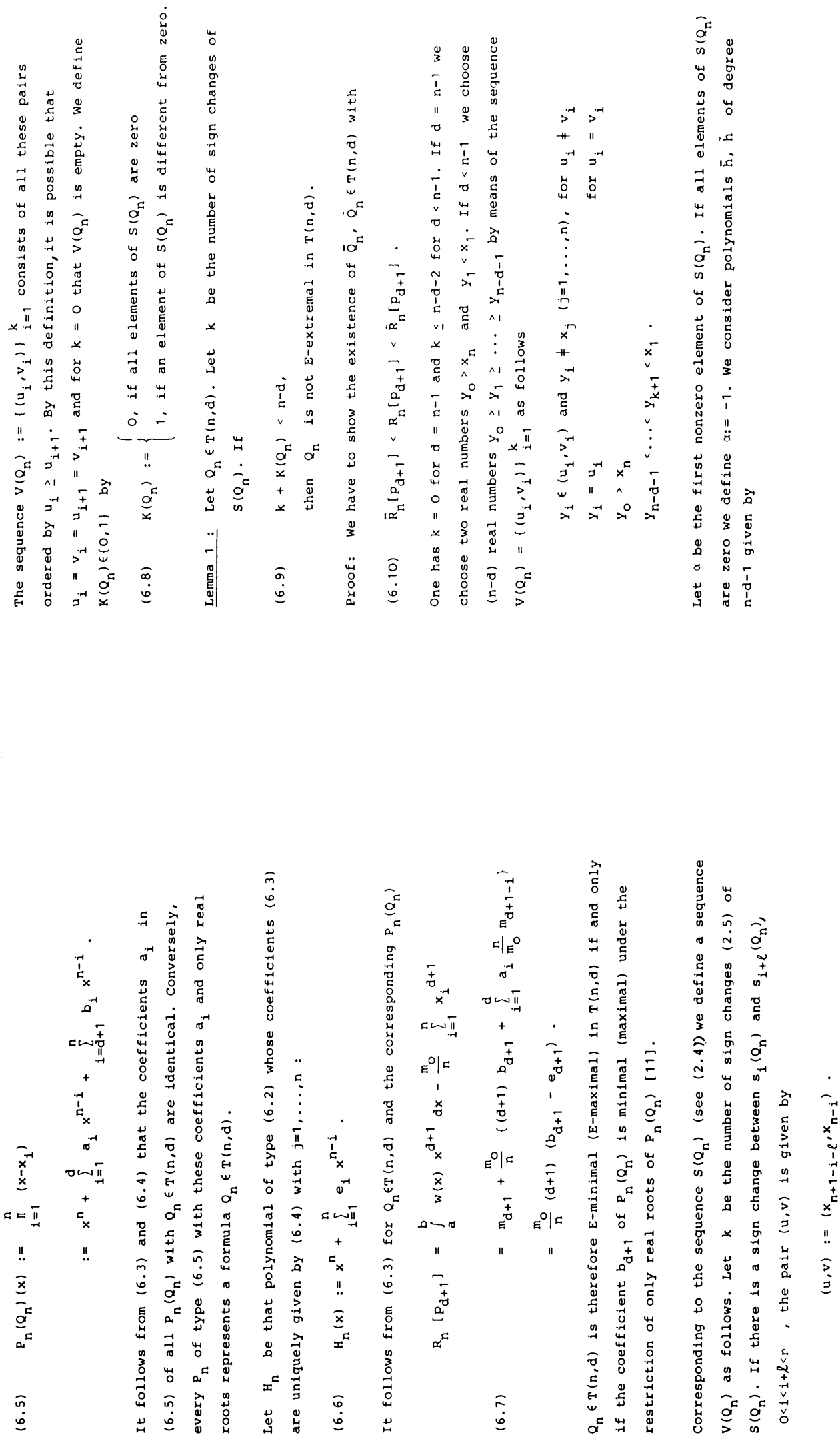

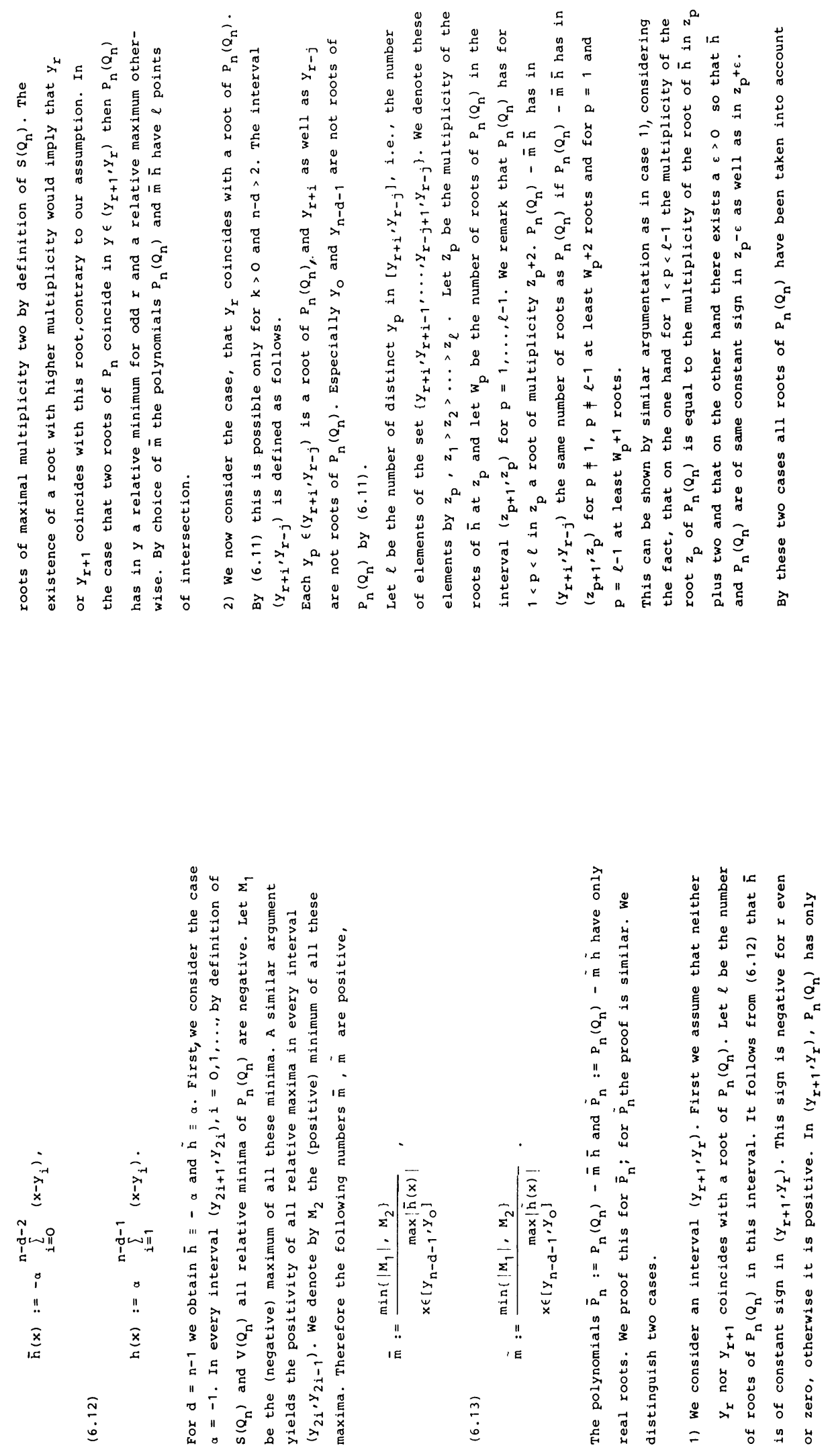

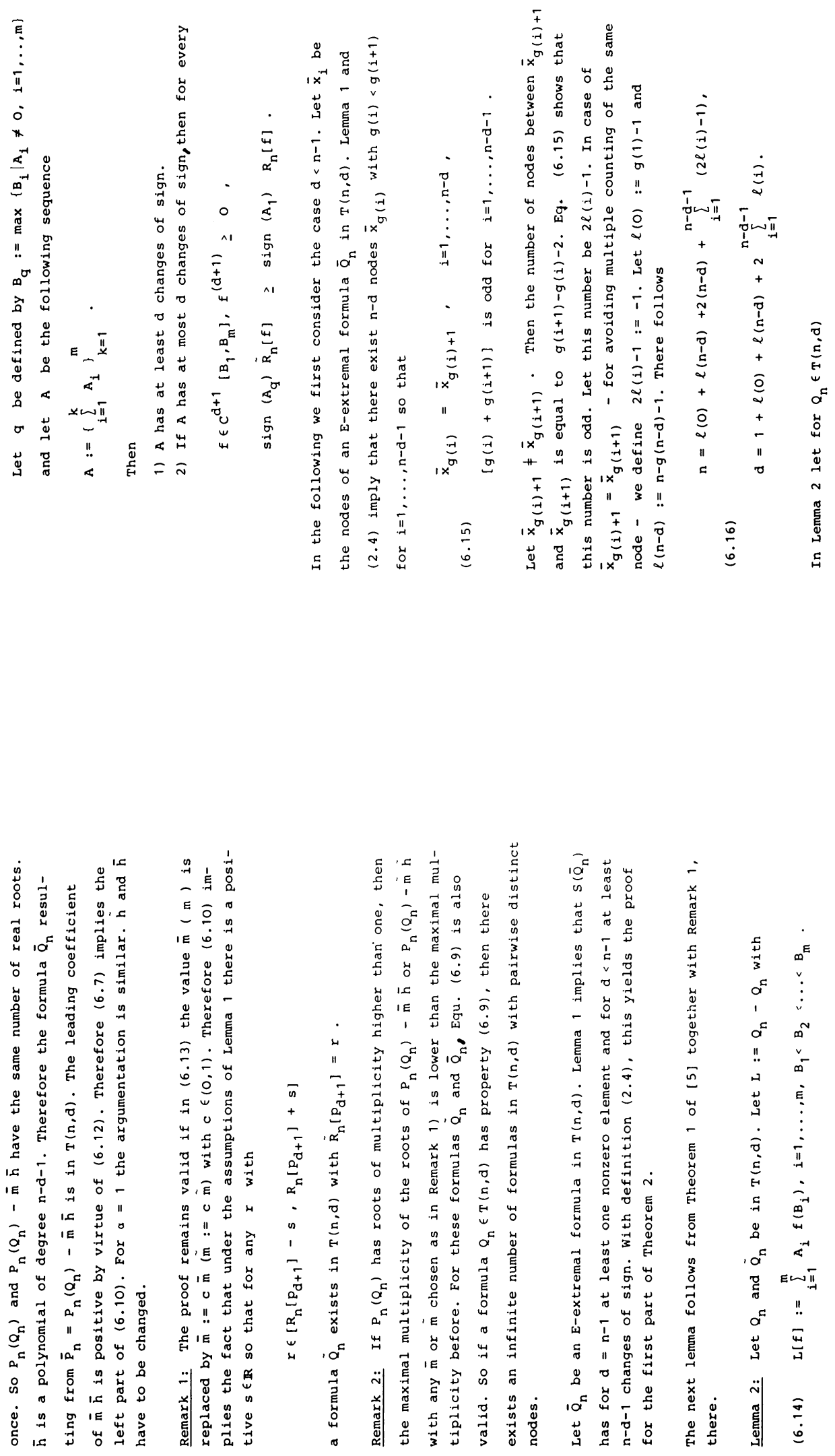

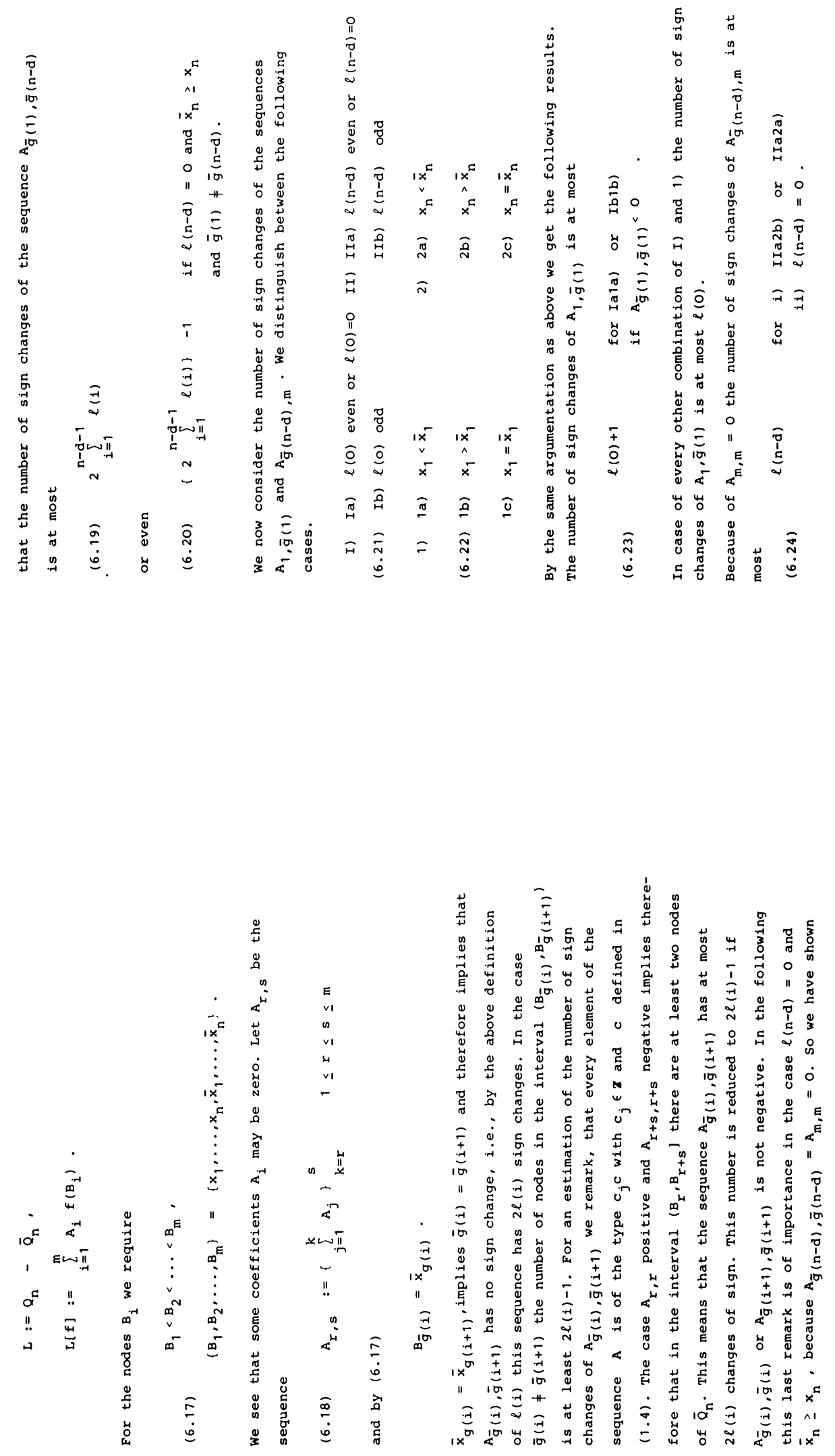

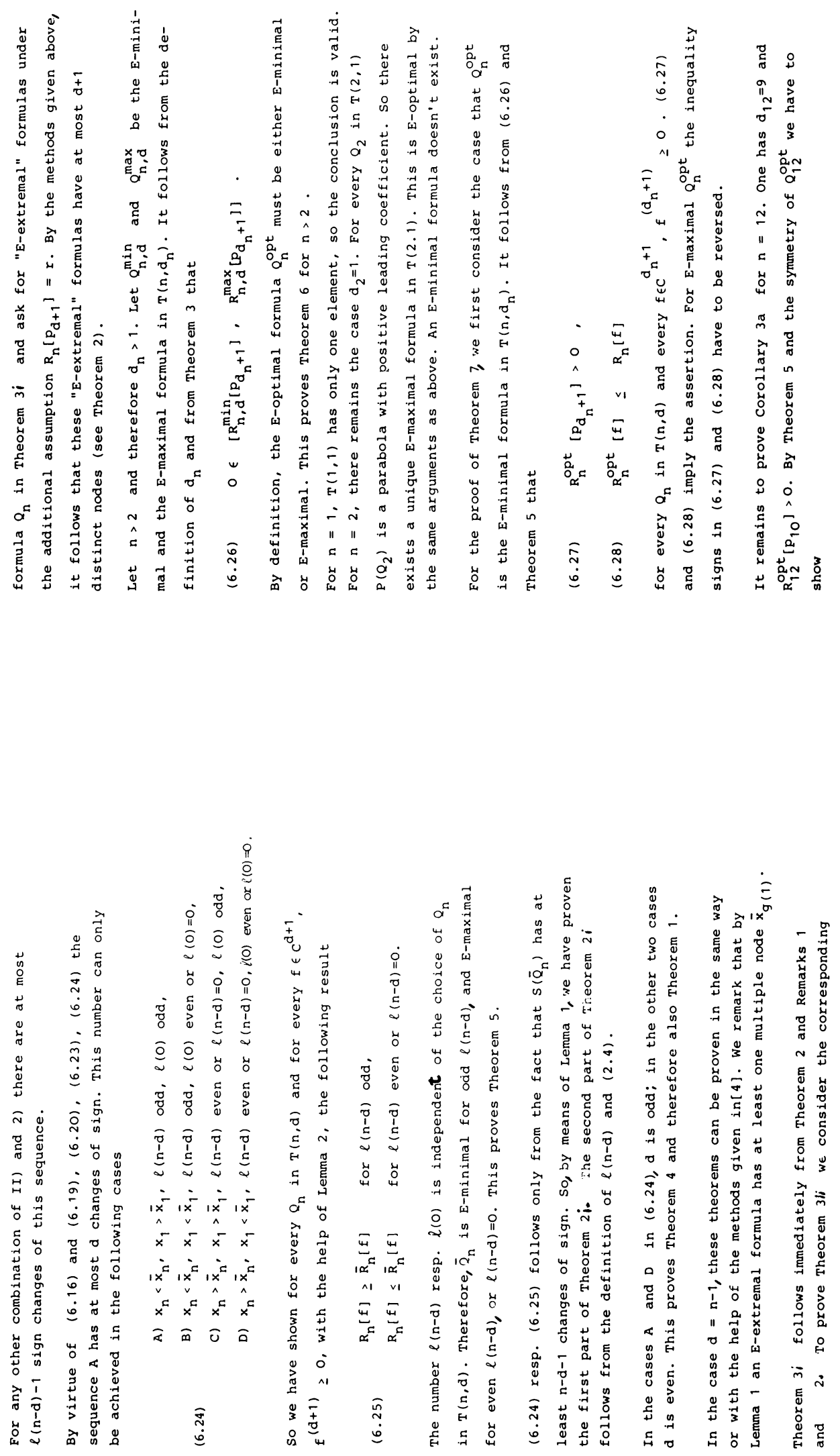


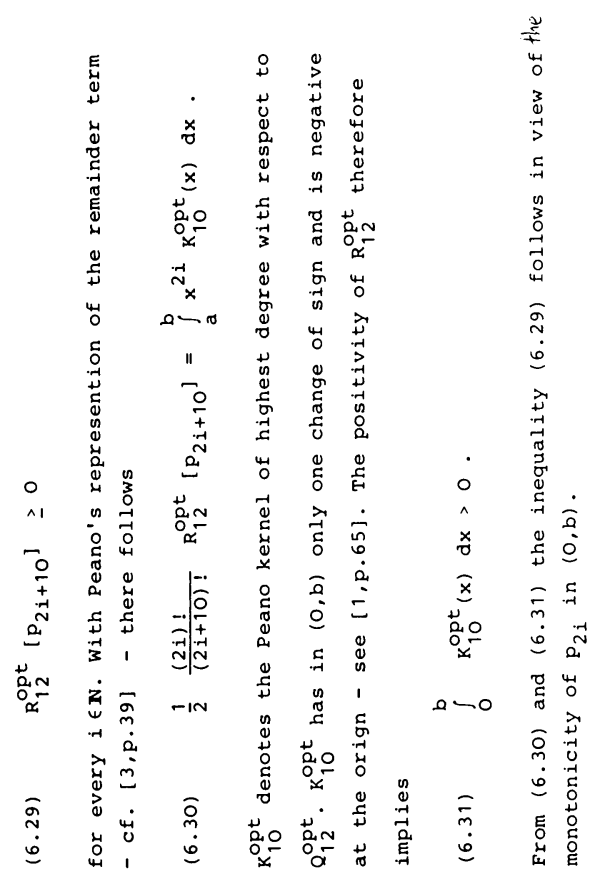

